\title{
The sums of the reciprocals of Fibonacci polynomials and Lucas polynomials
}

\author{
Zhengang $\mathrm{Wu}^{*}$ and Wenpeng Zhang
}

* Correspondence: sky.wzgfff@163. com

Department of Mathematics, Northwest University, Xi'an,

Shaanxi, P.R. China

\section{Abstract}

In this article, we consider infinite sums derived from the reciprocals of the Fibonacci polynomials and Lucas polynomials, and infinite sums derived from the reciprocals of the square of the Fibonacci polynomials and Lucas polynomials. Then applying the floor function to these sums, we obtain several new equalities involving the Fibonacci polynomials and Lucas polynomials.

Mathematics Subject Classification (2010): Primary, 11 B39.

Keywords: Fibonacci polynomials, Lucas polynomials, inequality, floor function

\section{Introduction}

For any variable quantity $x$, the Fibonacci polynomials $F_{n}(x)$ and Lucas polynomials $L_{n}$ $(x)$ are defined by $F_{n+2}(x)=x F_{n+1}(x)+F_{n}(x), n \geq 0$ with the initial values $F_{0}(x)=0$ and $F_{1}(x)=1 ; L_{n+2}(x)=x L_{n+1}(x)+L_{n}(x), n \geq 0$ with the initial values $L_{0}(x)=2$ and $L_{1}(x)=$ $x$. For $x=1$ we obtain the usual Fibonacci numbers and Lucas numbers. Let $\alpha=\frac{1}{2}\left(x+\sqrt{x^{2}+4}\right)$ and $\beta=\frac{1}{2}\left(x-\sqrt{x^{2}+4}\right)$, then from the properties of the second-order linear recurrence sequences we have

$$
F_{n}(x)=\frac{\alpha^{n}-\beta^{n}}{\sqrt{x^{2}+4}} \text { and } L_{n}(x)=\alpha^{n}+\beta^{n} .
$$

Various authors studied the properties of Fibonacci polynomials and Lucas polynomials, and obtained many interesting results, see [1-3]. Recently, several authors studied the infinite sums derived from the reciprocals of the Fibonacci numbers and Pell numbers, and obtained some important results. For example, Ohtsuka and Nakamura [4] studied the properties of the Fibonacci numbers, and proved the following conclusions:

$$
\begin{aligned}
& \left\lfloor\left(\sum_{k=n}^{\infty} \frac{1}{F_{k}}\right)^{-1}\right\rfloor=\left\{\begin{array}{l}
F_{n-2}, \quad \text { if } n \text { is even and } n \geq 2 ; \\
F_{n-2}-1, \text { if } n \text { is odd and } n \geq 1 .
\end{array}\right. \\
& \left\lfloor\left(\sum_{k=n}^{\infty} \frac{1}{F_{k}^{2}}\right)^{-1}\right\rfloor= \begin{cases}F_{n-1} F_{n}-1, \text { if } n \text { is even and } n \geq 2 ; \\
F_{n-1} F_{n,} & \text { if } n \text { is odd and } n \geq 1 .\end{cases}
\end{aligned}
$$

(c) 2012 Zhengang and Wenpeng; licensee Springer. This is an Open Access article distributed under the terms of the Creative Commons Attribution License (http://creativecommons.org/licenses/by/2.0), which permits unrestricted use, distribution, and reproduction in any medium, provided the original work is properly cited. 
Wenpeng and Tingting [5] studied the infinite sums derived from the Pell numbers, and obtained two similar results.

In this article, we considered infinite sums derived from the reciprocal of the Fibonacci polynomials and Lucas polynomials, and proved the following:

Theorem 1. For any positive integer $x$, we have

$$
\left\lfloor\left(\sum_{k=n}^{\infty} \frac{1}{F_{k}(x)}\right)^{-1}\right\rfloor=\left\{\begin{array}{l}
F_{n}(x)-F_{n-1}(x), \quad \text { if } n \text { is even and } n \geq 2 ; \\
F_{n}(x)-F_{n-1}(x)-1, \text { if } n \text { is odd and } n \geq 1 .
\end{array}\right.
$$

Theorem 2. For any positive integer $x$, we have

$$
\left\lfloor\left(\sum_{k=n}^{\infty} \frac{1}{F_{k}^{2}(x)}\right)^{-1}\right\rfloor= \begin{cases}x F_{n-1}(x) F_{n}(x)-1, & \text { if } n \text { is even and } n \geq 2 ; \\ x F_{n-1}(x) F_{n}(x), & \text { if } n \text { is odd and } n \geq 1\end{cases}
$$

Theorem 3. For any positive integer $x$, we have

$$
\left\lfloor\left(\sum_{k=n}^{\infty} \frac{1}{L_{k}(x)}\right)^{-1}\right\rfloor= \begin{cases}L_{n}(x)-L_{n-1}(x)-1, & \text { if } n \text { is even and } n \geq 2 ; \\ L_{n}(x)-L_{n-1}(x), & \text { if } n \text { is odd and } n \geq 3 .\end{cases}
$$

Theorem 4. For any positive integer $x \geq 2$, we have

$$
\left\lfloor\left(\sum_{k=n}^{\infty} \frac{1}{L_{k}^{2}(x)}\right)^{-1}\right\rfloor=\left\{\begin{array}{l}
x L_{2 n-1}(x)+1, \text { if } n \text { is even and } n \geq 2 ; \\
x L_{2 n-1}(x)-2, \text { if } n \text { is odd and } n \geq 3 .
\end{array}\right.
$$

If $x=1$ and $x=2$, then from our theorems we can deduce the conclusions of $[4,5]$. Especially, we also have the following:

Corollary 1 . For any positive integer $n$, we have the identities

$$
\left\lfloor\left(\sum_{k=n}^{\infty} \frac{1}{L_{k}}\right)^{-1}\right\rfloor=\left\{\begin{array}{l}
L_{n-2}-1, \text { if } n \text { is even and } n \geq 2 ; \\
L_{n-2}, \quad \text { if } n \text { is odd and } n \geq 1
\end{array}\right.
$$

Corollary 2. For any positive integer $n$, we have the identities

$$
\left\lfloor\left(\sum_{k=n}^{\infty} \frac{1}{P_{k}}\right)^{-1}\right\rfloor=\left\{\begin{array}{l}
P_{n}-P_{n-1}, \quad \text { if } n \text { is even and } n \geq 2 ; \\
P_{n}-P_{n-1}-1, \text { if } n \text { is odd and } n \geq 1 ;
\end{array}\right.
$$

Corollary 3. For any positive integer $n$, we have the identities

$$
\left\lfloor\left(\sum_{k=n}^{\infty} \frac{1}{P_{k}^{2}}\right)^{-1}\right\rfloor= \begin{cases}2 P_{n-1} P_{n}-1, & \text { if } n \text { is even and } n \geq 2 ; \\ 2 P_{n-1} P_{n}, & \text { if } n \text { is odd and } n \geq 1 .\end{cases}
$$

\section{Proof of theorems}

In this section, we shall complete the proof of our theorems. We shall prove only Theorems 1 and 2, and the other two theorems are proved similarly and omitted. First we prove Theorem 1. We consider the case that $n=2 m$ is even. At this time, Theorem 1 equivalent to 


$$
\frac{1}{F_{2 m}(x)-F_{2 m-1}(x)+1}<\sum_{k=2 m}^{\infty} \frac{1}{F_{k}(x)} \leq \frac{1}{F_{2 m}(x)-F_{2 m-1}(x)} .
$$

Now we prove that for any positive integers $x$ and $k \geq 1$,

$$
\frac{1}{F_{2 k}(x)}+\frac{1}{F_{2 k+1}(x)}<\frac{1}{F_{2 k}(x)-F_{2 k-1}(x)}-\frac{1}{F_{2 k+2}(x)-F_{2 k+1}(x)} .
$$

This inequality equivalent to

$$
\frac{F_{2 k-1}(x)}{F_{2 k}(x)\left(F_{2 k}(x)-F_{2 k-1}(x)\right)}>\frac{F_{2 k+2}(x)}{F_{2 k+1}(x)\left(F_{2 k+2}(x)-F_{2 k+1}(x)\right)},
$$

or

$$
\begin{aligned}
& F_{2 k-1}(x) F_{2 k+1}(x)\left(F_{2 k+2}(x)-F_{2 k+1}(x)\right) \\
& >F_{2 k}(x) F_{2 k+2}(x)\left(F_{2 k}(x)+F_{2 k-1}(x)\right),
\end{aligned}
$$

applying the identities

$$
\begin{aligned}
& F_{m}(x) F_{n}(x)=\frac{1}{x^{2}+4}\left(L_{m+n}(x)-(-1)^{n} L_{m-n}(x)\right), \\
& L_{m}(x) L_{n}(x)=L_{m+n}(x)+(-1)^{n} L_{m-n}(x), \\
& F_{m}(x) L_{n}(x)=F_{m+n}(x)+(-1)^{n} F_{m-n}(x)=F_{m+n}(x)-(-1)^{m} F_{n-m}(x),
\end{aligned}
$$

the inequality (2.2) equivalent to

$$
\begin{aligned}
& \left(L_{4 k}(x)+L_{2}(x)\right)\left(F_{2 k+2}(x)-F_{2 k+1}(x)\right) \\
& >\left(L_{4 k+2}(x)-L_{2}(x)\right)\left(F_{2 k}(x)-F_{2 k-1}(x)\right),
\end{aligned}
$$

or

$$
\begin{aligned}
& F_{2 k+4}(x)+2 F_{2 k+2}(x)-F_{2 k+1}(x) \\
& +F_{2 k}(x)-2 F_{2 k-1}(x)-F_{2 k-3}(x)>0 .
\end{aligned}
$$

It is easy to check that the inequality (2.3) holds for any positive integers $x$ and $k \geq 1$. So the inequality (2.2) is true. Using (2.2) repeatedly, we have

$$
\begin{aligned}
\sum_{k=2 m}^{\infty} \frac{1}{F_{k}(x)} & =\sum_{k=m}^{\infty}\left(\frac{1}{F_{2 k}(x)}+\frac{1}{F_{2 k+1}(x)}\right) \\
& <\sum_{k=m}^{\infty}\left(\frac{1}{F_{2 k}(x)-F_{2 k-1}(x)}-\frac{1}{F_{2 k+2}(x)-F_{2 k+1}(x)}\right) \\
& =\frac{1}{F_{2 m}(x)-F_{2 m-1}(x)} .
\end{aligned}
$$

On the other hand, we prove that for any positive integers $x$ and $k \geq 1$,

$$
\begin{aligned}
& \frac{1}{F_{2 k}(x)}+\frac{1}{F_{2 k+1}(x)} \\
& >\frac{1}{F_{2 k}(x)-F_{2 k-1}(x)+1}-\frac{1}{F_{2 k+2}(x)-F_{2 k+1}(x)+1} .
\end{aligned}
$$


The inequality (2.5) equivalent to

$$
\frac{F_{2 k+2}(x)+1}{F_{2 k+1}(x)\left(F_{2 k+2}(x)-F_{2 k+1}(x)+1\right)}>\frac{F_{2 k-1}(x)-1}{F_{2 k}(x)\left(F_{2 k}(x)-F_{2 k-1}(x)+1\right)}
$$

or

$$
\begin{aligned}
& L_{4 k+3}(x)-L_{4 k-1}(x)-F_{2 k+3}(x)-\left(x^{2}+3\right) F_{2 k+2}(x)+\left(2 x^{2}+8\right) F_{2 k+1}(x) \\
& +2 F_{2 k}(x)+\left(x^{2}+3\right) F_{2 k-1}(x)+F_{2 k-2}(x)-2 x^{2}-4>0 .
\end{aligned}
$$

It is easy to check that the inequality (2.6) holds for all positive integers $x$ and $k \geq 1$. So the inequality (2.5) is true. Using (2.5) repeatedly, we have

$$
\begin{aligned}
\sum_{k=2 m}^{\infty} \frac{1}{F_{k}(x)} & =\sum_{k=m}^{\infty}\left(\frac{1}{F_{2 k}(x)}+\frac{1}{F_{2 k+1}(x)}\right) \\
& >\sum_{k=m}^{\infty}\left(\frac{1}{F_{2 k}(x)-F_{2 k-1}(x)+1}-\frac{1}{F_{2 k+2}(x)-F_{2 k+1}(x)+1}\right) \\
& =\frac{1}{F_{2 m}(x)-F_{2 m-1}(x)+1} .
\end{aligned}
$$

Now the inequality (2.1) follows from (2.4) and (2.7).

Similarly, we can consider the case that $n=2 m+1$ is odd. Note that $F_{1}(x)-F_{0}(x)-$ $1=0$ and

$$
\sum_{k=1}^{\infty} \frac{1}{F_{k}(x)}=1+\frac{1}{x}+\frac{1}{x^{2}+1}+\cdots>1,
$$

So Theorem 1 is true if $m=0$. If $m \geq 1$, then our Theorem 1 equivalent to the inequality

$$
\frac{1}{F_{2 m+1}(x)-F_{2 m}(x)}<\sum_{k=2 m+1}^{\infty} \frac{1}{F_{k}(x)} \leq \frac{1}{F_{2 m+1}(x)-F_{2 m}(x)-1} .
$$

First we can prove that for any positive integers $x$ and $k \geq 1$,

$$
\begin{aligned}
& \frac{1}{F_{2 k+1}(x)}+\frac{1}{F_{2 k+2}(x)} \\
& <\frac{1}{F_{2 k+1}(x)-F_{2 k}(x)-1}-\frac{1}{F_{2 k+3}(x)-F_{2 k+2}(x)-1} .
\end{aligned}
$$

The inequality (2.9) equivalent to

$$
\frac{F_{2 k+3}(x)-1}{F_{2 k+2}(x)\left(F_{2 k+3}(x)-F_{2 k+2}(x)-1\right)}<\frac{F_{2 k}(x)+1}{F_{2 k+1}(x)\left(F_{2 k+1}(x)-F_{2 k}(x)-1\right)}
$$

or

$$
\begin{aligned}
& L_{4 k+5}(x)-L_{4 k+1}(x)-F_{2 k+5}(x)-F_{2 k+4}(x)-3 F_{2 k+3}(x)-F_{2 k+2}(x) \\
& -3 F_{2 k+1}(x)+F_{2 k}(x)-F_{2 k-1}(x)+F_{2 k-2}(x)+2 x^{2}+8>0
\end{aligned}
$$

It is easy to check that the inequality (2.10) holds for all positive integers $x$ and $k \geq 1$. So the inequality (2.9) is true. Using (2.9) repeatedly, we have 


$$
\begin{aligned}
\sum_{k=2 m+1}^{\infty} \frac{1}{F_{k}(x)} & =\sum_{k=m}^{\infty}\left(\frac{1}{F_{2 k+1}(x)}+\frac{1}{F_{2 k+2}(x)}\right) \\
& <\sum_{k=m}^{\infty}\left(\frac{1}{F_{2 k+1}(x)-F_{2 k}(x)-1}-\frac{1}{F_{2 k+3}(x)-F_{2 k+2}(x)-1}\right) \\
& =\frac{1}{F_{2 m+1}(x)-F_{2 m}(x)-1} .
\end{aligned}
$$

On the other hand, we prove that for any positive integers $x$ and $k \geq 1$,

$$
\frac{1}{F_{2 k+1}(x)}+\frac{1}{F_{2 k+2}(x)}>\frac{1}{F_{2 k+1}(x)-F_{2 k}(x)}-\frac{1}{F_{2 k+3}(x)-F_{2 k+2}(x)} .
$$

The inequality (2.12) equivalent to

$$
\frac{F_{2 k+3}(x)}{F_{2 k+2}(x)\left(F_{2 k+3}(x)-F_{2 k+2}(x)\right)}>\frac{F_{2 k}(x)}{F_{2 k+1}(x)\left(F_{2 k+1}(x)-F_{2 k}(x)\right)}
$$

or

$$
\begin{aligned}
& F_{2 k+5}(x)+2 F_{2 k+3}(x)-F_{2 k+2}(x) \\
& +F_{2 k+1}(x)-2 F_{2 k}(x)-F_{2 k-2}(x)>0 .
\end{aligned}
$$

It is easy to check that the inequality (2.13) holds for all positive integers $x$ and $k \geq 1$. So the inequality (2.12) is true. Using (2.12) repeatedly, we have

$$
\begin{aligned}
\sum_{k=2 m+1}^{\infty} \frac{1}{F_{k}(x)} & =\sum_{k=m}^{\infty}\left(\frac{1}{F_{2 k+1}(x)}+\frac{1}{F_{2 k+2}(x)}\right) \\
& >\sum_{k=m}^{\infty}\left(\frac{1}{F_{2 k+1}(x)-F_{2 k}(x)}-\frac{1}{F_{2 k+3}(x)-F_{2 k+2}(x)}\right) \\
& =\frac{1}{F_{2 m+1}(x)-F_{2 m}(x)} .
\end{aligned}
$$

Combining (2.11) and (2.14) we deduce the inequality (2.8). This proves Theorem 1.

Proof of Theorem 2. First we consider the case that $n=2 m$ is even. At this time, Theorem 2 equivalent to

$$
\frac{1}{x F_{2 m-1}(x) F_{2 m}(x)}<\sum_{k=2 m}^{\infty} \frac{1}{F_{k}^{2}(x)} \leq \frac{1}{x F_{2 m-1}(x) F_{2 m}(x)-1} .
$$

Now we prove that for any positive integers $x$ and $k \geq 1$,

$$
\frac{1}{F_{2 k}^{2}(x)}+\frac{1}{F_{2 k+1}^{2}(x)}>\frac{1}{x F_{2 k-1}(x) F_{2 k}(x)}-\frac{1}{x F_{2 k+1}(x) F_{2 k+2}(x)} .
$$

So the inequality (2.16) equivalent to

$$
\frac{F_{2 k+3}(x)}{F_{2 k+1}^{2}(x) F_{2 k+2}(x)}>\frac{F_{2 k-2}(x)}{F_{2 k-1}(x) F_{2 k}^{2}(x)}
$$

or

$$
\left(L_{4}(x)-2\right)\left(L_{4 k+2}(x)+L_{4 k}(x)\right)>0 .
$$


It is clear that the inequality (2.17) holds for all positive integers $x$ and $k \geq 1$. So the inequality (2.16) is true. Using (2.16) repeatedly, we have

$$
\begin{aligned}
\sum_{k=2 m}^{\infty} \frac{1}{F_{k}^{2}(x)} & =\sum_{k=m}^{\infty}\left(\frac{1}{F_{2 k}^{2}(x)}+\frac{1}{F_{2 k+1}^{2}(x)}\right) \\
& >\sum_{k=m}^{\infty}\left(\frac{1}{x F_{2 k-1}(x) F_{2 k}(x)}-\frac{1}{x F_{2 k+1}(x) F_{2 k+2}(x)}\right) \\
& =\frac{1}{x F_{2 m-1}(x) F_{2 m}(x)} .
\end{aligned}
$$

On the other hand, we prove that for any positive integers $x$ and $k \geq 1$,

$$
\begin{aligned}
& \frac{1}{F_{2 k}^{2}(x)}+\frac{1}{F_{2 k+1}^{2}(x)} \\
& <\frac{1}{x F_{2 k-1}(x) F_{2 k}(x)-1}-\frac{1}{x F_{2 k+1}(x) F_{2 k+2}(x)-1} .
\end{aligned}
$$

So the inequality (2.19) equivalent to

$$
\frac{F_{2 k+1}(x) F_{2 k+3}(x)-1}{F_{2 k+1}^{2}(x)\left(x F_{2 k+1}(x) F_{2 k+2}(x)-1\right)}<\frac{F_{2 k-2}(x) F_{2 k}(x)+1}{F_{2 k}^{2}(x)\left(x F_{2 k-1}(x) F_{2 k}(x)-1\right)},
$$

or

$$
\begin{aligned}
& 2 L_{8 k+6}(x)+4 L_{8 k+4}(x)-4 L_{8 k}(x)-2 L_{8 k-2}(x)+L_{4 k+8}(x) \\
& -6 L_{4 k+4}(x)-11 L_{4 k+2}(x)-11 L_{4 k}(x)-6 L_{4 k-2}(x)+L_{4 k-6}(x)>0 .
\end{aligned}
$$

It is clear that the inequality (2.20) holds for all positive integers $x$ and $k \geq 1$.

So the inequality (2.19) is true. Using (2.19) repeatedly, we have

$$
\begin{aligned}
\sum_{k=2 m}^{\infty} \frac{1}{F_{k}^{2}(x)} & =\sum_{k=m}^{\infty}\left(\frac{1}{F_{2 k}^{2}(x)}+\frac{1}{F_{2 k+1}^{2}(x)}\right) \\
& <\sum_{k=m}^{\infty}\left(\frac{1}{x F_{2 k-1}(x) F_{2 k}(x)-1}-\frac{1}{x F_{2 k+1}(x) F_{2 k+2}(x)-1}\right) \\
& =\frac{1}{x F_{2 m-1}(x) F_{2 m}(x)-1} .
\end{aligned}
$$

Now the inequality (2.15) follows from (2.18) and (2.21).

Similarly, we can consider the case that $n=2 m+1$ is odd. Note that $x F_{0}(x) F_{1}(x)=0$ and

$$
\sum_{k=1}^{\infty} \frac{1}{F_{k}^{2}(x)}=1+\frac{1}{x^{2}}+\cdots>1
$$

So Theorem 2 is true if $m=0$. If $m \geq 1$, then Theorem 2 equivalent to

$$
\frac{1}{x F_{2 m}(x) F_{2 m+1}(x)+1}<\sum_{k=2 m+1}^{\infty} \frac{1}{F_{k}^{2}(x)} \leq \frac{1}{x F_{2 m}(x) F_{2 m+1}(x)} .
$$


First we prove that for any positive integers $x$ and $k \geq 1$,

$$
\frac{1}{F_{2 k+1}^{2}(x)}+\frac{1}{F_{2 k+2}^{2}(x)}<\frac{1}{x F_{2 k}(x) F_{2 k+1}(x)}-\frac{1}{x F_{2 k+2}(x) F_{2 k+3}(x)} .
$$

So the inequality (2.23) equivalent to

$$
\frac{F_{2 k+4}(x)}{F_{2 k+2}^{2}(x) F_{2 k+3}(x)}<\frac{F_{2 k-1}(x)}{F_{2 k}(x) F_{2 k+1}^{2}(x)}
$$

or

$$
\left(L_{4}(x)-2\right)\left(L_{4 k+4}(x)+L_{4 k+2}(x)\right)>0 .
$$

It is clear that the inequality (2.24) is correct. So the inequality (2.23) is true.

Using (2.23) repeatedly, we have

$$
\begin{aligned}
\sum_{k=2 m+1}^{\infty} \frac{1}{F_{k}^{2}(x)} & =\sum_{k=m}^{\infty}\left(\frac{1}{F_{2 k+1}^{2}(x)}+\frac{1}{F_{2 k+2}^{2}(x)}\right) \\
& <\sum_{k=m}^{\infty}\left(\frac{1}{x F_{2 k}(x) F_{2 k+1}(x)}-\frac{1}{x F_{2 k+2}(x) F_{2 k+3}(x)}\right) \\
& =\frac{1}{x F_{2 m}(x) F_{2 m+1}(x)} .
\end{aligned}
$$

On the other hand, we prove that for any positive integers $x$ and $k \geq 1$,

$$
\begin{aligned}
& \frac{1}{F_{2 k+1}^{2}(x)}+\frac{1}{F_{2 k+2}^{2}(x)} \\
& >\frac{1}{x F_{2 k}(x) F_{2 k+1}(x)+1}-\frac{1}{x F_{2 k+2}(x) F_{2 k+3}(x)+1} .
\end{aligned}
$$

So the inequality (2.26) equivalent to

$$
\frac{F_{2 k+2}(x) F_{2 k+4}(x)+1}{F_{2 k+2}^{2}(x)\left(x F_{2 k+2}(x) F_{2 k+3}(x)+1\right)}>\frac{F_{2 k-1}(x) F_{2 k+1}(x)-1}{F_{2 k+1}^{2}(x)\left(x F_{2 k}(x) F_{2 k+1}(x)+1\right)}
$$

or

$$
\begin{aligned}
& 2 L_{8 k+10}(x)+4 L_{8 k+8}(x)-4 L_{8 k+4}(x)-2 L_{8 k+2}(x)-L_{4 k+10}(x) \\
& +6 L_{4 k+6}(x)+11 L_{4 k+4}(x)+11 L_{4 k+2}(x)+6 L_{4 k}(x)-L_{4 k-4}(x)>0 .
\end{aligned}
$$

It is clear that inequality (2.27) is correct. So the inequality (2.26) is true.

Using (2.26) repeatedly, we have

$$
\begin{aligned}
\sum_{k=2 m+1}^{\infty} \frac{1}{F_{k}^{2}(x)} & =\sum_{k=m}^{\infty}\left(\frac{1}{F_{2 k+1}^{2}(x)}+\frac{1}{F_{2 k+2}^{2}(x)}\right) \\
& >\sum_{k=m}^{\infty}\left(\frac{1}{x F_{2 k}(x) F_{2 k+1}(x)+1}-\frac{1}{x F_{2 k+2}(x) F_{2 k+3}(x)+1}\right) \\
& =\frac{1}{x F_{2 m}(x) F_{2 m+1}(x)+1} .
\end{aligned}
$$

Now the inequality (2.22) follows from (2.25) and (2.28). This proves Theorem 2. 


\section{Acknowledgements}

The authors express their gratitude to the referee for very helpful and detailed comments. This work is supported by the N.S.F. of P.R.China (11071194)

\section{Authors' contributions}

ZW proposed if we could obtain some similarly results in [45], and proved a part of the theorems. Wu Zhengang obtained the theorems and completed the proof. All authors read and approved the final manuscript.

\section{Competing interests}

The authors declare that they have no competing interests.

Received: 15 February 2012 Accepted: 11 June 2012 Published: 11 June 2012

\section{References}

1. Falcón, S, Plaza, Á: On the Fibonacci k-numbers, chaos, solitons \& fractals. 32, 1615-1624 (2007)

2. Ma, R, Zhang, W: Several identities involving the Fibonacci numbers and Lucas numbers. Fibon Q. 45, 164-170 (2007)

3. $Y \mathrm{Y}, \mathrm{Y}$, Zhang, W: Some identities involving the Fibonacci polynomials. Fibon Q. 40, 314-318 (2002)

4. Ohtsuka, H, Nakamura, S: On the sum of reciprocal Fibonacci numbers. Fibon Q. 46/47, 153-159 (2009)

5. Zhang, W, Wang, T: The infinite sum of reciprocal Pell numbers. Appl Math Comput. 218, 6164-6167 (2012). doi:10.1016/j.amc.2011.11.090

doi:10.1186/1029-242X-2012-134

Cite this article as: Wu and Zhang: The sums of the reciprocals of Fibonacci polynomials and Lucas polynomials. Journal of Inequalities and Applications 2012 2012:134.

\section{Submit your manuscript to a SpringerOpen ${ }^{\odot}$} journal and benefit from:

Convenient online submission

- Rigorous peer review

- Immediate publication on acceptance

- Open access: articles freely available online

- High visibility within the field

- Retaining the copyright to your article

Submit your next manuscript at $\gg$ springeropen.com 